\title{
Evaluation of Tip Performance by XE-100 Atomic Force Microscopy (AFM)
}

\author{
Jun S. Lee*, Joonhyung Kwon**, Sang-il Park**, and Changmo Sung* \\ * Center for Advanced Materials, University of Massachusetts, Lowell, MA 01854 \\ ** PSIA Corporation, Seocho-dong 1600-3, Seoul 137-070, Korea
}

Atomic Force microscopy (AFM) is common to use for inorganic and organic materials in terms of the interaction force between atoms. By means of these phenomena, AFM image is a convolution of a tip shape and a surface geometry. Tip characterization is a key factor to obtain a true image of sample without a tip deconvolution. There are several ways to check the tip conditions by the reference sample [1] and software in terms of a blind reconstruction algorithm [2]. This theory is able to construct the effect of tip deconvolution by erosion, subtracted from the original image, and dilation, added to the scanned image [3].

High performance lab-sized XE-100 AFM (PSIA Corp.) was used as a tool to scan a sample by $5 \mu \mathrm{m}$ x $5 \mu \mathrm{m}$ [4]. The scanned sample was a polymeric material which was a copolymer of amphiphilic decyl esters of the amino acids L-tyrosine (DELT) and tyrosine amid after 24 hours. The scanned image of sample is shown Fig. 1 in 2D view with scan rate 0.5Hz and Fig. 2 in 3 dimensional view based on the topography. A non-contact ultrasharp silicon cantilever (NSC15 series) with Al coating on the laser-reflection side was used in this study. The dimensions of cantilever are $125 \mu \mathrm{m} \times 35 \mu \mathrm{m}$ $\mathrm{x} 4 \mu \mathrm{m}$. A spring force constant is $40 \mathrm{~N} / \mathrm{m}$ and a resonant frequency is typically $325 \mathrm{kHz}$. The squareshaped used and new tips were utilized to find out the effect of tip convolution how the used tip is performed properly to validate the convolution in the image analysis tool (XEI). The configuration of tip estimation parameters was as follows; image size in pixel, $512 \times 512$, and tip size in pixel, 25 . After conducting the tip estimation, the tip shape was generated Fig. 3 in $3 \mathrm{D}$ view of the reconstructed tip shape. Fig. 4 shows a side view of the reconstructed tip based on the method of tip estimation. In addition, the certainty map [2] was produced through tip deconvolution.

The comparison of an original image from topographical information with the deconvoluted image was well matched each other in the XEI. In accordance with both the bead type of sample and the tip scanning mechanism, the effect of dilation and erosion was dramatically reduced. Therefore, the condition of tip end was easily identified by using the cross-sectional view of the reconstructed tip shape against a real tip. In Fig. 5, the certainty map shows the confidence of the reconstructed sample image through the tip deconvolution. It is concluded that the tip estimation and deconvolution can be easily obtained and compared with the convoluted and the deconvoluted images.

\section{References}

[1] H.-Y. Nie and N. S. McIntyre, Langmuir, 17 (2001) 432.

[2] J. S. Villarrubia, J. Res. Natl. Inst. Stand. Technol., 102 (1997) 425.

[3] S. Dongmo, et al., J. Vac. Sci. Technol. B 14 (1996) 1552

[4] Joonhyung Kwon, et al., Review of Scientific Instruments, October (2002).

[5] The sample was provided by Kenneth A. Marx, Department of Chemistry, University of

Massachusetts. 


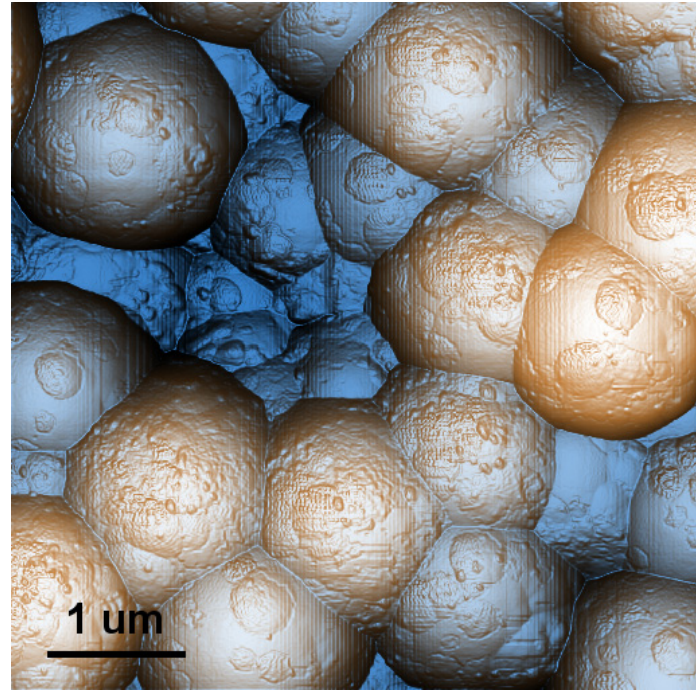

FIG. 1. Non-contact topography image.

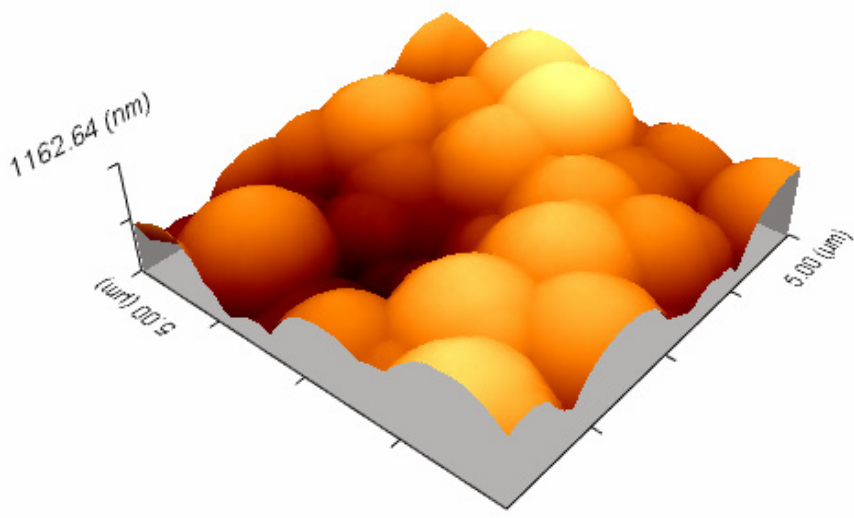

FIG. 2. 3D view based on the topography.
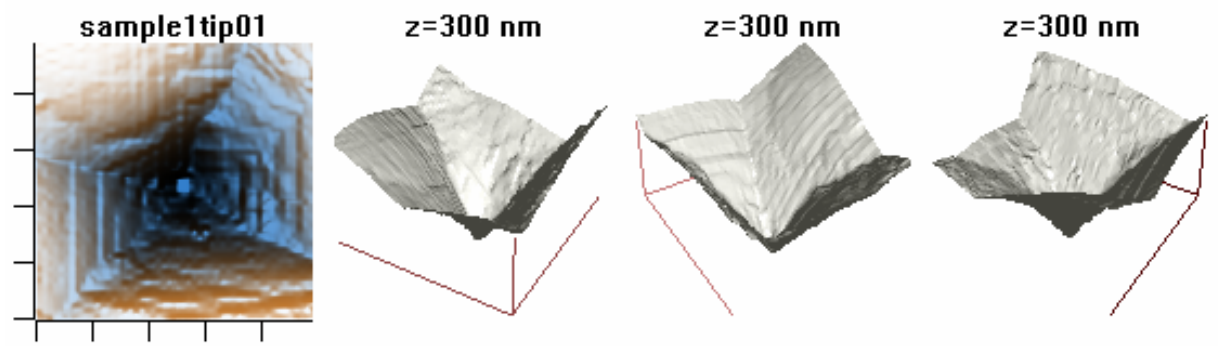

$100 \mathrm{~nm} / \mathrm{div}$

FIG. 3. Top view and 3D view of silicon tip by performing the tip estimation.

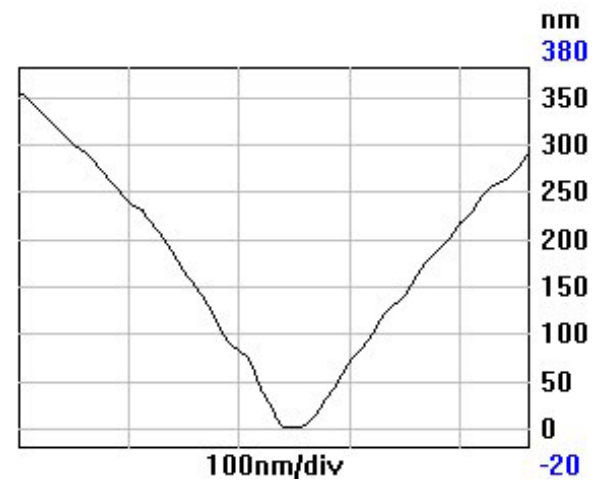

FIG. 4. 2D view of tip after tip estimation.

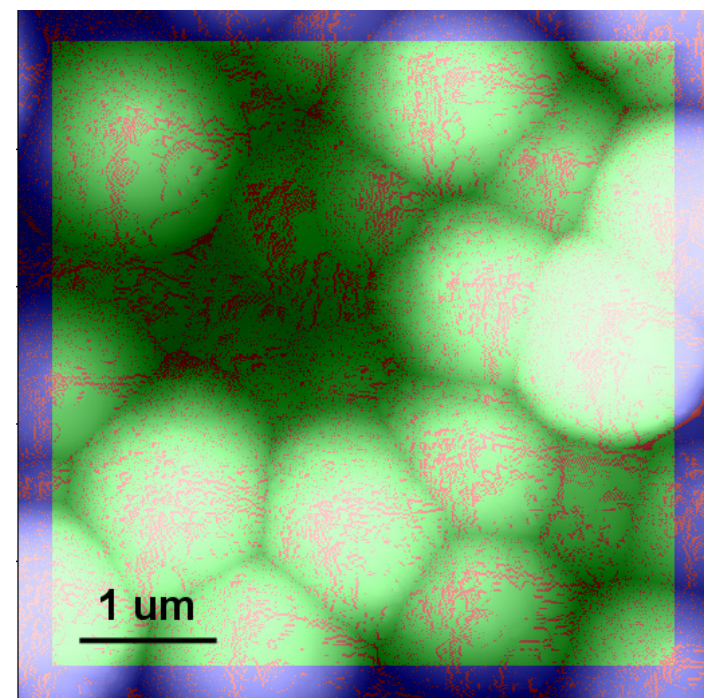

FIG. 5. Certainty map after tip deconvolution. 